\title{
Influence of Air Staging on the Dynamics of a Precessing Vortex Core in a Dual-Swirl Gas Turbine Model Combustor
}

\author{
Christoph M. Arndt ${ }^{1}$, Michael Stöhr ${ }^{2}$, Michael J. Severin ${ }^{3}$, Claudiu Dem ${ }^{4}$, Wolfgang Meier ${ }^{5}$ \\ German Aerospace Center (DLR), Institute of Combustion Technology, 70569 Stuttgart, Germany
}

\begin{abstract}
Detailed laser diagnostic and optical measurements to study temperature, major species concentration, velocity field and flame shape, have been carried out in a partially premixed dual-swirl gas turbine model combustor (GTMC) at atmospheric pressure using methane as fuel. The GTMC features separate air plenums for the inner and outer air stream, thus allowing control of the air split ratio between the inner and outer air stream. In the current study, flames with a thermal power of $22.5 \mathrm{~kW}$ and a global equivalence ratio of $\varphi=0.63$ have been studied for air split ratios $L$ between 1.2 and 2.0 , with $L=1.6$ corresponding to equal pressure loss across both swirlers. Temperature and major species concentrations were measured with laser Raman scattering, the velocity field with particle image velocimetry (PIV) and the flame shape and position with $\mathrm{OH}^{*}$ chemiluminescence. For all air split ratios, the flame did not exhibit strong thermo-acoustic oscillations, such that the global heat release rate did not vary with time. However, due to a precessing vortex core (PVC) that was present for all operating conditions, strong variations in the local heat release distribution of the flame could be observed. The frequency of the PVC was at a constant Strouhal number, which was based on the air mass flow through the inner air nozzle, but was independent of the air split ratio. The Strouhal number for the PVC was $\mathrm{Sr}_{\mathrm{nr}}=\mathbf{0 . 7 8}$ for the non-reacting case and $\mathrm{Sr}_{\mathrm{r}}=\mathbf{0 . 8 5}$ for the reacting case. The current paper focuses on (a) providing a detailed data set for the validation of numerical simulations of this combustor and (b) on the influence of the air split ratio on the dynamics of the PVC.
\end{abstract}

$\begin{array}{llll} & & \text { Nomenclature } & \\ \mathrm{D} & =\text { diameter } & \mathrm{CL} & =\text { Chemiluminescence } \\ f & =\text { focal length } & \mathrm{GT} & =\text { gas turbine } \\ \mathrm{f} / & =\text { f-number } & \mathrm{GTMC} & =\text { gas turbine model combustor } \\ \mathrm{f} & =\text { frequency } & \mathrm{IRO} & =\text { Intensified relay optics } \\ l & =\text { length scale } & \mathrm{IRZ} & =\text { inner recirculation zone } \\ L & =\text { air split ratio } & \mathrm{ORZ} & =\text { outer recirculation zone } \\ \dot{m} & =\text { mass flow } & \mathrm{PMT} & =\text { Photomultiplyer tube } \\ P_{t h} & =\text { thermal power } & \mathrm{PTU} & =\text { Programmable Timing Unit } \\ \mathrm{u}, \mathrm{v}, \mathrm{w} & =\text { radial, axial, tangential velocity component } & \mathrm{PVC} & =\text { precessing vortex core } \\ |\mathrm{v}| & =\text { total flow velocity } & \mathrm{PIV} & =\text { Particle Image Velocimetry } \\ \mathrm{X}, \mathrm{y}, \mathrm{z} & =\text { Cartesian coordinates } & \mathrm{dt} & =\text { pulse separation time } \\ X & =\text { mole fraction } & (\cdot)_{s t} & =\text { stoichiometric } \\ \alpha & =\text { phase angle } & (\cdot)_{i} & =\text { inner } \\ \varphi & =\text { equivalence ratio } & (\cdot)_{o} & =\text { outer } \\ \xi & =\text { mixture fraction } & (\cdot)_{r} & =\text { reacting } \\ \tau & =\text { particle relaxation time } & (\cdot)_{n r} & =\text { non-reacting } \\ \mathrm{Sr} & =\text { Strouhal number } & (\cdot)^{\prime} & =\text { average } \\ \mathrm{St} & =\text { Stokes number } & (\cdot)^{\prime} & =\text { standard deviation }\end{array}$

\footnotetext{
${ }^{1}$ Research Associate, Combustion Diagnostics Department, christoph.arndt@dlr.de

${ }^{2}$ Research Associate, Combustion Diagnostics Department

${ }^{3}$ Research Associate, Combustion Diagnostics Department

${ }^{4}$ Research Associate, Combustion Diagnostics Department

${ }^{5}$ Department Head, Combustion Diagnostics Department
} 


\section{Introduction}

$\mathrm{M}^{-10}$ ODERN gas turbine (GT) combustors often are equipped with swirl burners for lean premixed or partially premixed operation. While lean-premixed combustors feature excellent emission levels, operational restrictions arise from their susceptibility to thermo-acoustic instabilities. In this case, the unsteady heat release couples with one or more acoustic modes of the combustor. Despite significant research effort and progress in this field, reliable prediction of frequencies and amplitudes of thermo-acoustic oscillations remains a challenging task. This is due to the complex interaction between heat release, flow field and acoustic modes of the combustor. Mminor geometrical modifications of the combustor or small changes in the operating or boundary conditions can lead to drastic changes in the stability of the system, such as transition from attached to detached flames or flames with and without precessing vortex cores $(\mathrm{PVCs})^{1,2}$. The occurrence of hydrodynamic instabilities like PVCs, vortex shedding, or other shear layer instabilities ${ }^{3-8}$ further complicates the situation. For example, PVCs can influence the mixing ${ }^{7,9-11}$, or can cause flame roll-up and local extinction ${ }^{6,12}$. Furthermore, PVCs can interact with thermo-acoustic oscillations ${ }^{5,13}$. In several GT combustors, PVCs were observed both in the reacting and in the non-reacting flow field $^{6,14-16}$, while for other configurations a PVC was present in the non-reacting flow field, but was not present in certain, but not necessarily all, reacting flow fields ${ }^{17-22}$.

Good progress for understanding the fundamental properties of instationary phenomena in swirl-stabilized combustors has been achieved by the use of GT model combustors, which feature several properties of practical engines, such as self-excited oscillations, swirl-induced vortex breakdown, hydrodynamic instabilities, and timedependent premixing, while providing well controlled operating and boundary conditions at reasonable costs. Furthermore, such model combustors can be equipped with combustion chambers with very good optical access in order to apply laser based measurement techniques to determine important quantities such as temperature, flow field and species concentration with high temporal and spatial resolution.

In the current study, a dual-swirl gas turbine model combustor (GTMC) was investigated experimentally with detailed laser-based and optical techniques. The burner features two separate air plenums for each air flow, allowing the air split ratio between the inner and the outer air flow to be set exactly. The paper focuses on the detailed experimental characterization of an operating condition without self-excited thermo-acoustic oscillations, but with a precessing vortex core. The flame shape was measured by $\mathrm{OH}^{*}$ chemiluminescence imaging, the simultaneous major species concentration, mixture fraction, and temperature by laser Raman scattering and the three-component planar velocity field by stereoscopic Particle Image Velocimetry. Furthermore, the pressure oscillations in both air plenums and in the combustion chamber were measured by dynamic pressure probes. The effect of the air split ratio on the non-reacting and reacting flow fields as well as on the flame shape are addressed, and the dynamics of the observed precessing vortex core and its impact on the flame behavior were studied.

\section{Experimental Setup}

\section{A. SFB Dual Swirl Burner}

A schematic of the combustor can be seen in Figure 1. Details of the burner geometry can be found in the literature $^{23,24}$, and only a brief overview is given here. The design is based on previously studied dual-swirl-burner configurations, but features several improvements to the boundary conditions. Most importantly, the burner features two swirlers with separate plenum chambers. Thus, the air flow to each plenum can be controlled independently, such that the air split ratio between the inner and outer nozzle can be set exactly. Here, the air split ratio $L$ is defined as the ratio of the mass flow through the outer and the inner swirler:

$$
L=\frac{\dot{m}_{\text {air }, o}}{\dot{m}_{\text {air }, i}}
$$

An air split ratio of $L=1.6$, which is approximately the ratio of the nozzle exit areas, results in similar bulk velocities and equal pressure drop across both swirlers ${ }^{23}$. The theoretical swirl number is $\mathrm{S}_{0}=1.06$ for the outer swirler and $S_{i}=0.73$ for the inner swirler. Fuel is supplied to the inner nozzle through a feed system built into the splitter wall between the nozzles. The fuel flows through a circle of 60 holes, which are $0.5 \mathrm{~mm}$ in diameter and are located on the inner wall surface $12 \mathrm{~mm}$ below the nozzle exit. In this configuration, the flow from the inner nozzle is partially premixed before the onset of combustion. The flames are enclosed by a square combustion chamber ( $89 \mathrm{~mm} \times 89 \mathrm{~mm}$ cross section, $112 \mathrm{~mm}$ high) comprised of four fused silica plates held by steel frames. The frames are mounted to four posts in the corners and to the bottom and top of the combustion chamber. The combustion chamber offers very good optical access and it is equipped with several ports for pressure probes. A nearly identical setup is available for liquid fuels ${ }^{25}$. Further, the design allows for several identical burners to be combined into a combustor array, in order to investigate cross-flame interactions ${ }^{26,27}$. Previous studies have focused on the feedback 
mechanism of the thermo-acoustic cycle ${ }^{23}$, the variations of the thermo-chemical state throughout the acoustic cycle ${ }^{24}$ and the impact of the material temperature on thermoacoustic properties of the burner ${ }^{28}$.

All flow rates were metered by electromechanical mass flow controllers (Brooks type 5851S for $\mathrm{CH}_{4}$ and type 5853S for each air flow) and additionally monitored by calibration-standard Coriolis flow meters (Siemens SITRANS F C MASS 2100 DI 3 for $\mathrm{CH}_{4}$ and Siemens SITRANS F C MASS 2100 DI 15 for each air flow) with an accuracy of approximately $1.5 \%$. A wide range of parameters of equivalence ratio, thermal power, and air split ratio has been examined. However, this paper focuses on a set of operating conditions with fixed thermal power and global equivalence ratio, but different air split ratios $L$. An overview of the operating conditions studied in this paper is shown in Table 1 . The coordinate system has its origin $(\mathrm{x}, \mathrm{y}, \mathrm{z}=0 \mathrm{~mm})$ in the center of the burner exit plane.

The flow field was measured with particle image velocimetry (PIV), the heat release zone with $\mathrm{OH}^{*}$ chemiluminescence and the pressure oscillations with microphone probes in the combustion chamber and in the air plenums. Furthermore, point-wise single shot Raman measurements have been performed at the base-line case with an air split ratio of $L=1.6$ in order to gain information about the major species and temperature distribution within the combustion chamber and to provide validation data for numerical simulations.

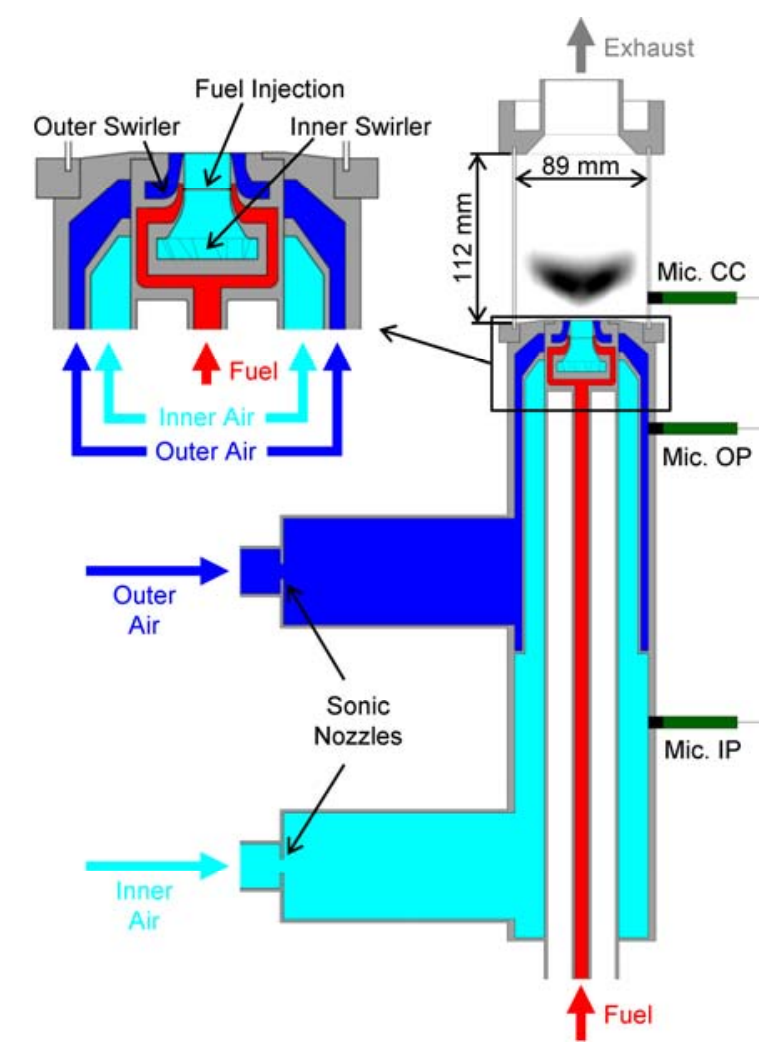

Figure 1. Schematic of the SFB dual-swirl burner.

\section{B. Stereoscopic Particle Image Velocimetry (S-PIV)}

The three-component velocity field in the measurement plane was acquired with Stereoscopic Particle Image Velocimetry (S-PIV) at a repetition rate of $5 \mathrm{~Hz}$. Titanium dioxide $\left(\mathrm{TiO}_{2}\right)$ particles were added to the flow and illuminated with a frequency doubled dual-head Nd:YAG laser (New Wave Solo 120) with a pulse energy of $120 \mathrm{~mJ}$ at $532 \mathrm{~nm}$ and a pulse separation time of $\mathrm{dt}_{\mathrm{nr}}=10 \mu \mathrm{s}$ for the non-reacting flows and $\mathrm{dt}_{\mathrm{r}}=8 \mu \mathrm{s}$ for the reacting flows. The laser beam was formed into a vertical light sheet and aligned with the central plane of the combustion chamber. The beam width in the test section was approximately $1 \mathrm{~mm}$. Mie scattering of the particles was recorded with a pair of CCD cameras (LaVision Imager Intense, 1376 x 1040 pixels), which were operated in a double-frame mode. The timing of the experiment was controlled with a programmable timing unit (LaVision PTU 9). The cameras were mounted at an angle of $20^{\circ}$ relative to the laser light sheet and equipped with wide-angle lenses $(f=16 \mathrm{~mm}$, set to $\mathrm{f} / 2$ ) and band-pass filters $(532 \pm 5 \mathrm{~nm})$ to suppress background luminosity of the flame. The distance between the camera lenses and the measurement plane was $200 \mathrm{~mm}$. Scheimpflug adapters were used to align the focal plane of the cameras with the laser light sheet.

An infrared filter was used to protect the cameras from thermal radiation of the burner. A total of 1200 single shot particle pairs were recorded for each operating condition and the velocity fields were calculated from the particle image pairs using a multi-scale cross-correlation PIV algorithm (LaVision DaVis 8.4): The final size of the interrogation windows was $12 \times 12$ pixels with a 50\% window overlap, resulting in an in-plane resolution of $1.1 \mathrm{~mm} \times 1.1 \mathrm{~mm}$ and a vector spacing of $0.55 \mathrm{~mm}$. The field of view spanned an area $-39 \mathrm{~mm}<\mathrm{x}<39 \mathrm{~mm}$ and $0.5 \mathrm{~mm}<\mathrm{z}<105 \mathrm{~mm}$.

With an accuracy of the peak-finding algorithm of \pm 0.1 pixel the maximum random uncertainty of the in-plane instantaneous velocities is $\pm 1.2 \mathrm{~m} / \mathrm{s}$. The seeding particles had a relaxation time $\tau \approx 5 \times 10^{-6} \mathrm{~s}$, the strongest observed local velocity differences were $\Delta \mathrm{v} \approx 40 \mathrm{~m} / \mathrm{s}$ and occurred over a length scale of $l \approx 10 \mathrm{~mm}$. This resulted in a Stokes number of $S t=(\tau \cdot \Delta v) / l \approx 0.02$ and thus velocity errors due to particle slip are considered negligible. With a stereoscopic viewing angle of $20^{\circ}$ relative to the measurement plane, the uncertainty of out-of-plane velocity is approximately three times higher than in-plane uncertainty ${ }^{29}$. 


\begin{tabular}{ccccccc}
\hline $\begin{array}{c}L \\
{[-]}\end{array}$ & $\begin{array}{c}\dot{m}_{\text {air }, i} \\
{[\mathrm{~g} / \mathrm{min}]}\end{array}$ & $\begin{array}{c}\dot{m}_{\text {air }, o} \\
{[\mathrm{~g} / \mathrm{min}]}\end{array}$ & $\begin{array}{c}\dot{m}_{\text {air,CH}} \\
{[\mathrm{g} / \mathrm{min}]}\end{array}$ & $\begin{array}{c}\mathrm{P}_{\text {th }} \\
{[\mathrm{kW}]}\end{array}$ & $\begin{array}{c}\varphi_{\text {global }} \\
{[-]}\end{array}$ & $\begin{array}{c}\xi_{\text {global }} \\
{[-]}\end{array}$ \\
\hline 1.2 & 334 & 401 & 27 & 22.5 & 0.63 & 0.0355 \\
1.6 & 283 & 452 & 27 & 22.5 & 0.63 & 0.0355 \\
2.0 & 245 & 490 & 27 & 22.5 & 0.63 & 0.0355 \\
\hline
\end{tabular}

Table 1. Operating Conditions. $\varphi_{\text {global }}$ and $\xi_{\text {global }}$ are the global equivalence ratio and mixture fraction.

\section{OH* Chemiluminescence (CL)}

$\mathrm{OH}^{*}$ chemiluminescence was measured simultaneously to the velocity fields using a LaVision Imager Intense camera in combination with a LaVision IRO image intensifier. The intensifier was equipped with a fast UV lens (Cerco, $f=45 \mathrm{~mm}, \mathrm{f} / 1.8$ ) and a bandpass filter centered around $310 \mathrm{~nm}$ to collect $\mathrm{OH}^{*}$ chemiluminescence in the $(1,0)$ and $(1,1)$ band.

\section{Laser Raman Scattering}

Single-shot laser Raman scattering was applied for pointwise measurements of the concentrations of the major species $\left(\mathrm{O}_{2}, \mathrm{~N}_{2}, \mathrm{CH}_{4}, \mathrm{H}_{2}, \mathrm{CO}, \mathrm{CO}_{2}, \mathrm{H}_{2} \mathrm{O}\right)$ and temperature. A detailed description of the measurement system can be found $\mathrm{in}^{30,31}$ and only a brief overview is given here. A flash-lamp pumped dye laser (Candela LFDL 20, wavelength $489 \mathrm{~nm}$, pulse energy $\approx 1.8 \mathrm{~J}$, pulse length $\approx 3 \mu \mathrm{s}$ ) was operated at a repetition rate of $5 \mathrm{~Hz}$. The laser beam was focused into the combustion chamber, resulting in a measurement volume of $0.6 \mathrm{~mm}$ length and $0.6 \mathrm{~mm}$ diameter. The Raman signal was collected using an achromatic lens $(\mathrm{D}=80 \mathrm{~mm}, f=160 \mathrm{~mm})$ and imaged onto the entrance slit of a spectrograph (SPEX 1802, $f=1 \mathrm{~m}$, slit width $2 \mathrm{~mm}$, dispersion $\approx 0.5 \mathrm{~nm} / \mathrm{mm}$ ). The dispersed and spatially separated signals from the different species were detected using individual photomultiplier tubes (PMTs) for each species in the focal plane of the spectrograph and sampled using boxcar integrators. The species number densities were derived from the measurement signals using calibration measurements and the temperature was derived from the total number density using the ideal gas law. Simultaneous detection of all major species within each laser pulse allowed the determination of the instantaneous mixture fraction ${ }^{32}$. The mixture fraction was calculated according to the method of Bilger et al. ${ }^{33}$ and is defined as the ratio of mass originating from the fuel stream (all $\mathrm{C}$ and $\mathrm{H}$ atoms) to the total mass.

Measurements were performed at a total of 100 measurement locations at $\mathrm{z}=8,10,15,20,30,40,50,60,70$ and $80 \mathrm{~mm}$ at radial locations between $-3<\mathrm{x}<27 \mathrm{~mm}$. Measurement locations with $\mathrm{z}<8 \mathrm{~mm}$ and $\mathrm{x}>27 \mathrm{~mm}$ were not possible due to clipping of the solid angle of the detection optics.

The measurement locations are also shown as white closed symbols in Figure 4. A total of 400 single shot measurements was performed at each measurement location.

Regarding the measurement uncertainties, it has to be distinguished between systematic errors resulting from the calibration procedure and statistical errors resulting from statistics (shot noise) of the detected Raman photons in a single-shot measurement. Systematic uncertainties were $\pm 3-4 \%$ for temperature and mixture fraction and $\pm 3-5 \%$ for mole fractions of $\mathrm{O}_{2}, \mathrm{H}_{2} \mathrm{O}, \mathrm{CO}_{2}$ and $\mathrm{CH}_{4}$. For $\mathrm{H}_{2}$ and $\mathrm{CO}$ the measurement error was $\pm 20 \%$ at a mole fraction of 0.01 due to low concentration within the flame. Statistical uncertainties were approximately $30 \%$ larger than stated by Meier et al. ${ }^{31}$ due to lower pulse energy applied in the current measurements. Typical statistical uncertainties for a single shot measurement were $3-3.5 \%$ for the temperature and mixture fraction, $4 \%$ for $\mathrm{H}_{2} \mathrm{O}$ and $9 \%$ for $\mathrm{O}_{2}$ and $\mathrm{CO}_{2}$ in the exhaust gas. More details can be found in references ${ }^{31,34}$.

\section{E. Acoustic Measurements}

Pressure oscillations in the combustion chamber and in the two air plenums were measured using calibrated microphone probes (Brüel \& Kjær, type 4939), with a sampling rate of $50 \mathrm{kHz}$. The microphone probes were calibrated for frequencies up to $2000 \mathrm{~Hz}$. The pressure power spectrum at each location was computed by slicing the long-duration pressure signal into one-second segments, and calculating the power spectrum for each segment. Afterwards, the spectra of the segments were averaged, resulting in a frequency resolution of $1 \mathrm{~Hz}$. No additional filtering or smoothing of the raw signals or the frequency spectra was performed. Acoustic modes leading to an asymmetric pressure distribution in the combustion chamber, such as transversal thermo-acoustic modes or asymmetric flow instabilities like precessing vortex cores (PVCs) could be detected by calculating the frequency spectrum of the difference signal of two micropone probes that were mounted on opposite sides of the combustion chamber, but at the same axial position. 


\section{Results and Discussion}

\section{A. Characterization of the Baseline Case}

Before the influence of the air split ratio on the flow and flame is discussed, a detailed analysis of the baseline case is presented in order to gain an overview of the flow field and flame behavior in the current experiment. Figure 2 shows the mean and standard deviation of the $\mathrm{OH}^{*}$ chemiluminescence (CL) distribution as well as the Abeldeconvoluted $\mathrm{OH}^{*} \mathrm{CL}$ distribution for the reference operating condition $\left(L=1.6, \varphi_{\text {global }}=0.63, \mathrm{P}_{\mathrm{th}}=22.5 \mathrm{~kW}\right)$. Since $\mathrm{OH}^{*} \mathrm{CL}$ is a line-of-sight integrated measurement technique, spatially resolved information is difficult to draw from those measurements. However, since the here examined flames are rotationally symmetric, Abeldeconvolution can be applied to gain information on the average flame shape within a plane. The flame exhibits a Vlike conical shape with the main flame zone (represented by the region of highest $\mathrm{OH}^{*} \mathrm{CL}$ intensities) between $\mathrm{z}=25 \mathrm{~mm}$ and $\mathrm{z}=50 \mathrm{~mm}$. A strong increase in the $\mathrm{OH}^{*} \mathrm{CL}$ signal can be seen at $\mathrm{z}=8 \mathrm{~mm}$, however, the flame base is further upstream, approximately $4.5 \mathrm{~mm}$ downstream of the burner exit plane. This can be seen more clearly in the Abel-deconvoluted $\mathrm{OH}^{*} \mathrm{CL}$ distribution, and will be discussed below. Fluctuations of the $\mathrm{OH}^{*} \mathrm{CL}$ intensity, as visualized by the standard deviation of the $\mathrm{OH}^{*}$ signal, can be seen starting from $\mathrm{z}=4.5 \mathrm{~mm}$. The regions of the highest standard deviation are between $z=15 \mathrm{~mm}$ and $\mathrm{z}=45 \mathrm{~mm}$, thus approximately $5 \mathrm{~mm}$ upstream of the main region of the mean flame zone.

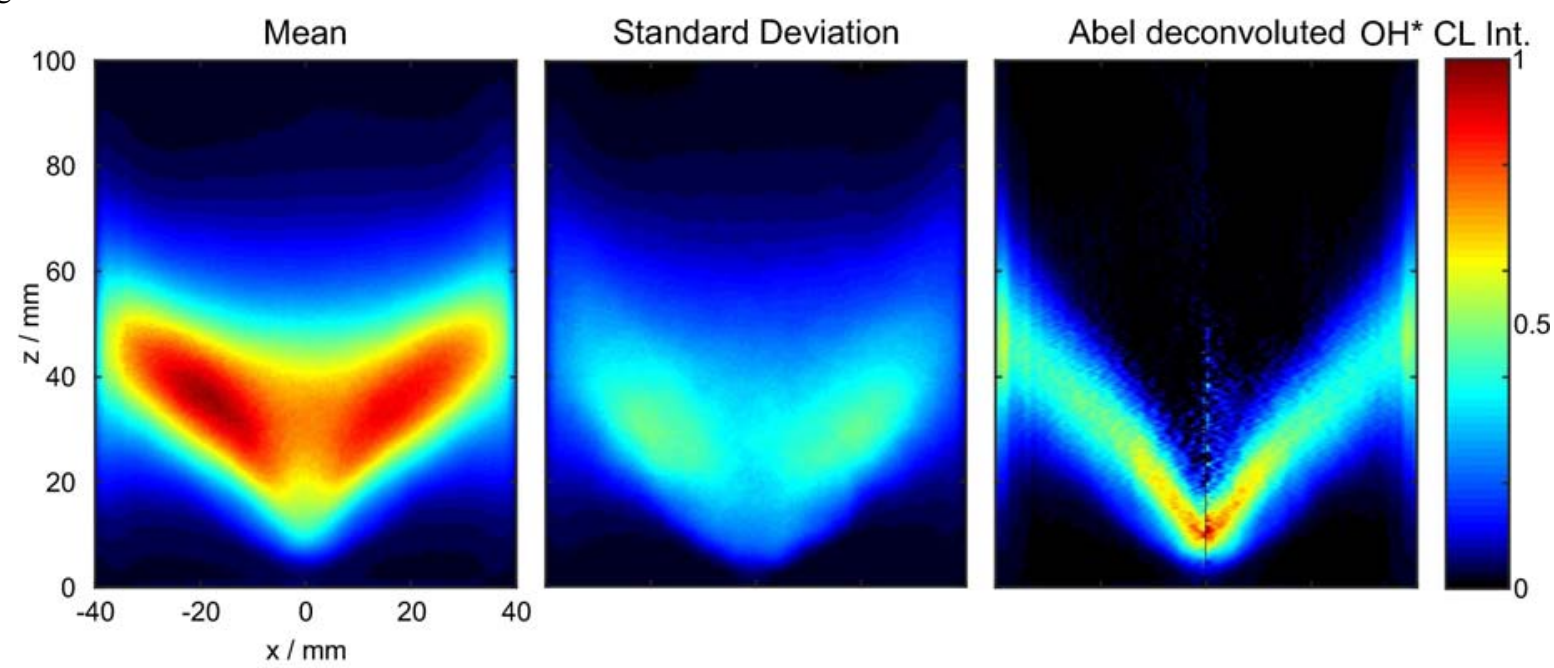

Figure 2. Mean (left), standard deviation (middle) and Abel-deconvolution (right) of the normalized $\mathrm{OH}^{*}$ chemiluminescence distribution for the reference operating condition $\left(L=1.6, \varphi_{\text {global }}=0.63\right.$, $P_{\text {th }}=22.5 \mathrm{~kW}$ ).

The Abel-deconvoluted $\mathrm{OH}^{*} \mathrm{CL}$ distribution shows that the flame root is approximately $4.5 \mathrm{~mm}$ downstream of the burner exit plane. Due to the V-shape of the flame and the radial weighting of the integrated $\mathrm{OH}^{*} \mathrm{CL}$ mean images, the flame base, which is close to the symmetry axis of the flame, is better distinguishable in the Abeldeconvoluted images. It is to note that interference from the red-hot glowing combustion chamber posts leads to increased intensity of the Abel-deconvoluted images near the combustion chamber walls. The high intensity variations on the symmetry axis are artifacts of the image processing algorithm. In the Abel-deconvoluted image, it is also clearly visible that the flame exhibits a V-like shape, with the highest local intensities occurring between $z=7.5 \mathrm{~mm}$ and $\mathrm{z}=30 \mathrm{~mm}$. Again, due to the radial weighting of line-of-sight integrated imaging techniques, the maximum of the flame zone appears to be further downstream.

An overview of the velocity field for the baseline case is given in Figure 3. Color coded are the mean values of the axial $(\bar{u})$ and radial $(\bar{v})$ velocity components, and overlaid are the sreamlines. The tangential velocity component $(\bar{w})$ is not shown here. The flame exhibits the typical behavior of swirl-stabilized flames with strong inner (IRZ) and outer (ORZ) recirculation zones, divided by a high-velocity inflow-region. The inner recirculation zone exhibits a "heart"-shape and extends to axial position up to $z=83 \mathrm{~mm}$. This particular shape of the recirculation zone stems from the influence of a vortex structure that forms at the combustion chamber outlet, known as exhaust tube vortex ${ }^{6}$. The core of this vortex structure can be seen at $\mathrm{z}>50 \mathrm{~mm}$ in the mean 2D-distribution of the axial velocity component. The lower boundary of the IRZ lies within the nozzle and thus is not accessible with optical measurement techniques. The radial extend of the IRZ at the burner exit is approximately $-2.5 \mathrm{~mm}<\mathrm{x}<2.5 \mathrm{~mm}$. 
The much weaker outer recirculation zone can be seen in the corners of the combustion chamber up to axial positions $\mathrm{z}<30 \mathrm{~mm}$ and at radial locations $|\mathrm{x}|>30 \mathrm{~mm}$. Although the bulk inflow velocities for the inner and outer airflow have the same nominal value, the inflow of the inner swirler exhibits higher peak values close to the nozzle. This is due to the inner recirculation zone that extends back into the inner air inlet, and thus blocking a part of the inflow, resulting in higher inflow velocities at the outer periphery of the inner air flow.
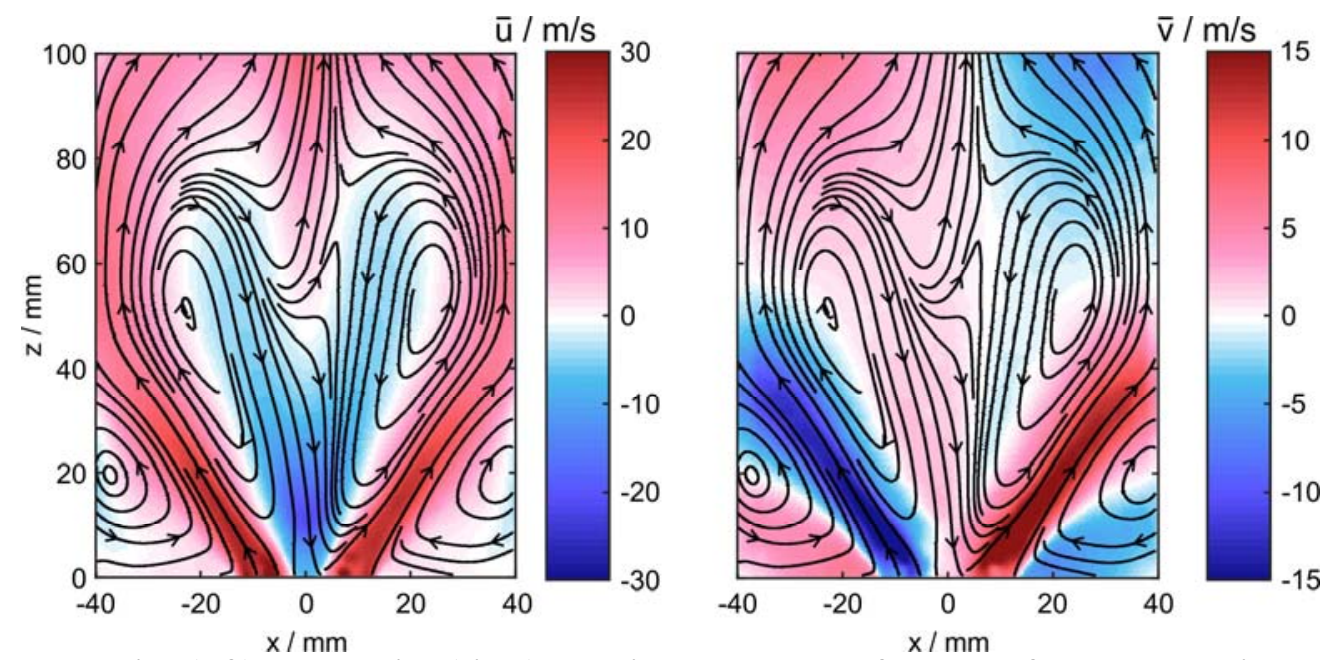

Figure 3. Mean axial (left) and radial (right) velocity components for the reference operating condition $\left(\mathrm{L}=\mathbf{1 . 6}, \varphi_{\text {global }}=0.63, \mathrm{P}_{\mathrm{th}}=22.5 \mathrm{~kW}\right)$. Overlaid are the streamlines.

The two inflows from the inner and outer nozzle can also be descerned in the 2D-plot of the mean axial velocity component $\bar{u}$. Here, a region of very low axial velocity can be seen at $|\mathrm{x}|=10 \mathrm{~mm}$ and $\mathrm{z}<5 \mathrm{~mm}$. The inflow is also clearly visible as a region of high velocities, extending up to $\mathrm{z}=55 \mathrm{~mm}$.

In technically premixed combustion, the flame stabilization is not only influenced by the flow field, but also by the thermo-chemical state of the flow. The flame front requires conditions that enable fast flame chemistry and a flow-field with locally low strain rates. From a chemical point of view, near-stoichiometric mixtures with elevated mixing temperatures provide ideal locations for the flame stabilization. In order to further assess the thermochemical state of the flame, Figure 4 shows the mean 2D-distributions of the Reynolds-averaged mixture fraction, the $\mathrm{CH}_{4}$ mole fraction, and the temperature. The two-dimensional plots were interpolated between the point-wise measurement locations (white close symbols) using the software "Origin 9.1". Due to the fact that the outer flow from the nozzle was pure air and the flow from the inner nozzle was partially premixed, significant spatial and temporal variations in the mixture fraction are expected due to finite mixing times and turbulence.

The mixture fraction can be used as a marker for the mixing process of the fuel with the air. The highest mixture fractions occur on the centerline of the burner $(x=0 \mathrm{~mm})$ at $z=8 \mathrm{~mm}$. Here, mixture fractions up to $\bar{\xi}=0.077$ occur, which is more than twice the global mixture fraction $\xi_{\text {global }}=0.0355$ and almost 1.5 times the stoichiometric mixture fraction $\xi_{s t}=0.055$. However, fuel and air mix quickly, and for $\mathrm{z} \geq 15 \mathrm{~mm}$ only lean mixtures with $\bar{\xi}<\xi_{s t}$ can be observed. At $z=40 \mathrm{~mm}$, the mean value for the mixture fraction is $\bar{\xi}=0.0395$, which is higher than the global mixture fraction $\xi_{\text {global }}=0.0355$. However, the mixture fraction decreases with increasing radius, and the leanest mixture fractions for $\mathrm{z}=40 \mathrm{~mm}$ are expected at $\mathrm{x}>27 \mathrm{~mm}$, and thus outside the field of view of the Raman measurements.

The $\mathrm{CH}_{4}$ mole fraction is a marker for the penetration depth of the fuel as well as for its mixing and consumption. The highest $\mathrm{CH}_{4}$ mole fractions are $\bar{X}_{C H 4}=0.11$ and occur at $\mathrm{x}=7 \mathrm{~mm}$ and z=8 mm, at the lowest measurement location. The highest $\mathrm{CH}_{4}$ mole fractions do not conincide with the highest mixture fractions, which occur at the symmetry axis of the burner. This could be due to burned gas that is transported upstream in the IRZ to the fuel nozzle and mixes with the inflowing fresh gas at this location. The fuel mixing and consumption process is quite fast, already at $\mathrm{z}=12 \mathrm{~mm}$, the highest occurring $\mathrm{CH}_{4}$ mole fractions are only $\bar{X}_{C H 4}=0.07$. At $\mathrm{z}=50 \mathrm{~mm}$, all fuel is consumed and the $\mathrm{CH}_{4}$ mole fraction remains zero. The decrease in $\bar{X}_{C H 4}$ with downstream distance correlates well with the flame shape that was measured with $\mathrm{OH}^{*} \mathrm{CL}$ (see Figure 2), meaning that the regions of the reaction zone correlate well with the regions of decreasing fuel mole fraction. 

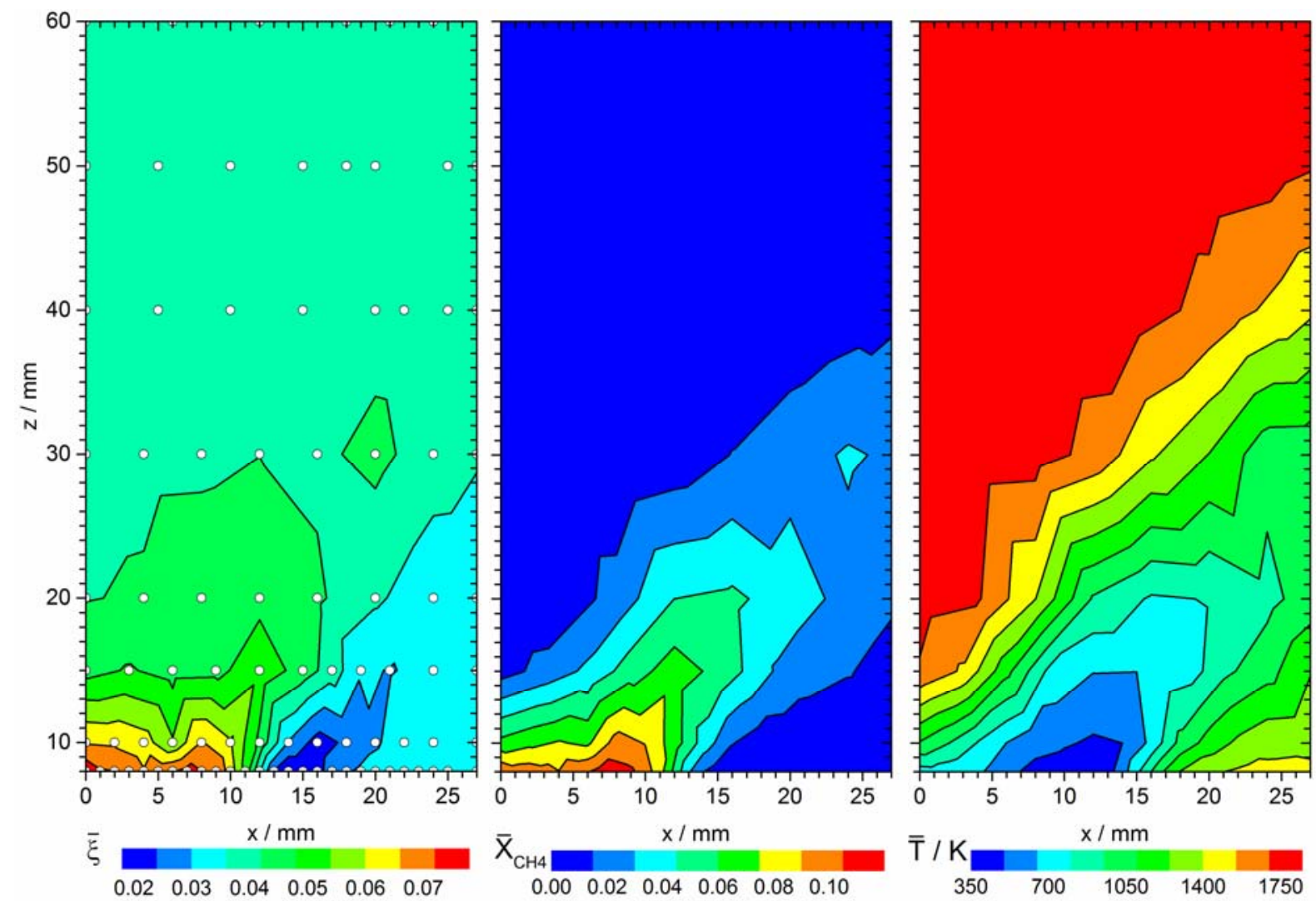

Figure 4. Mean mixture fraction, $\mathrm{CH}_{4}$ mole fraction and temperature field for the reference operating condition $\left(L=1.6, \varphi_{\text {global }}=0.63, P_{\text {th }}=22.5 \mathrm{~kW}\right)$.

The mean temperatures in the inflow region gradually increase with increasing axial location and reaction process. The highest mean temperatures occurred in the IRZ at $z=50 \mathrm{~mm}$ and $\mathrm{x}=10 \mathrm{~mm}$. A high temperature region can also be seen at $x>22 \mathrm{~mm}$ and $\mathrm{z}<10 \mathrm{~mm}$ in the ORZ where hot products are transported back and mix with the incoming fresh gas.

\section{B. Influence of the Air Split Ratio on Flame Shape, Flow Field, and Acoustics}

An overview of the different flame shapes for the here studied air split ratios $L$ is shown in Figure 5. The top row shows the mean $\mathrm{OH}^{*} \mathrm{CL}$ intensity and the bottom row shows the Abel-deconvoluted mean OH* CL images. Color coded is the $\mathrm{OH}^{*} \mathrm{CL}$ intensity. For all examined air split ratios, the flame features a V-shape and stabilizes between $2 \mathrm{~mm}$ and $7 \mathrm{~mm}$ downstream of the nozzle. The flame root is shifted further downstream with decreasing air split ratio $L$. Since fuel is only injected into the inner air stream, a decreasing are split ratio correlates with a decreasing mixture fraction and increasing flow velocities in the inner air flow. The shear layers between the inner and outer air stream become more pronounced when increasing or decreasing the air split ratio from its base value $L=1.6$, as will be discussed below. As described above, a finite mixing time is needed in order to achieve ideal mixing conditions for the flame, i.e. near-stoichiometric mixtures. Furthermore, elevated temperatures due to recirculation of hot products and low strain rates favor flame-stabilization. However, based on the $\mathrm{OH}^{*} \mathrm{CL}$ images alone, the reason for the different flame stabilization heights cannot be determined. 

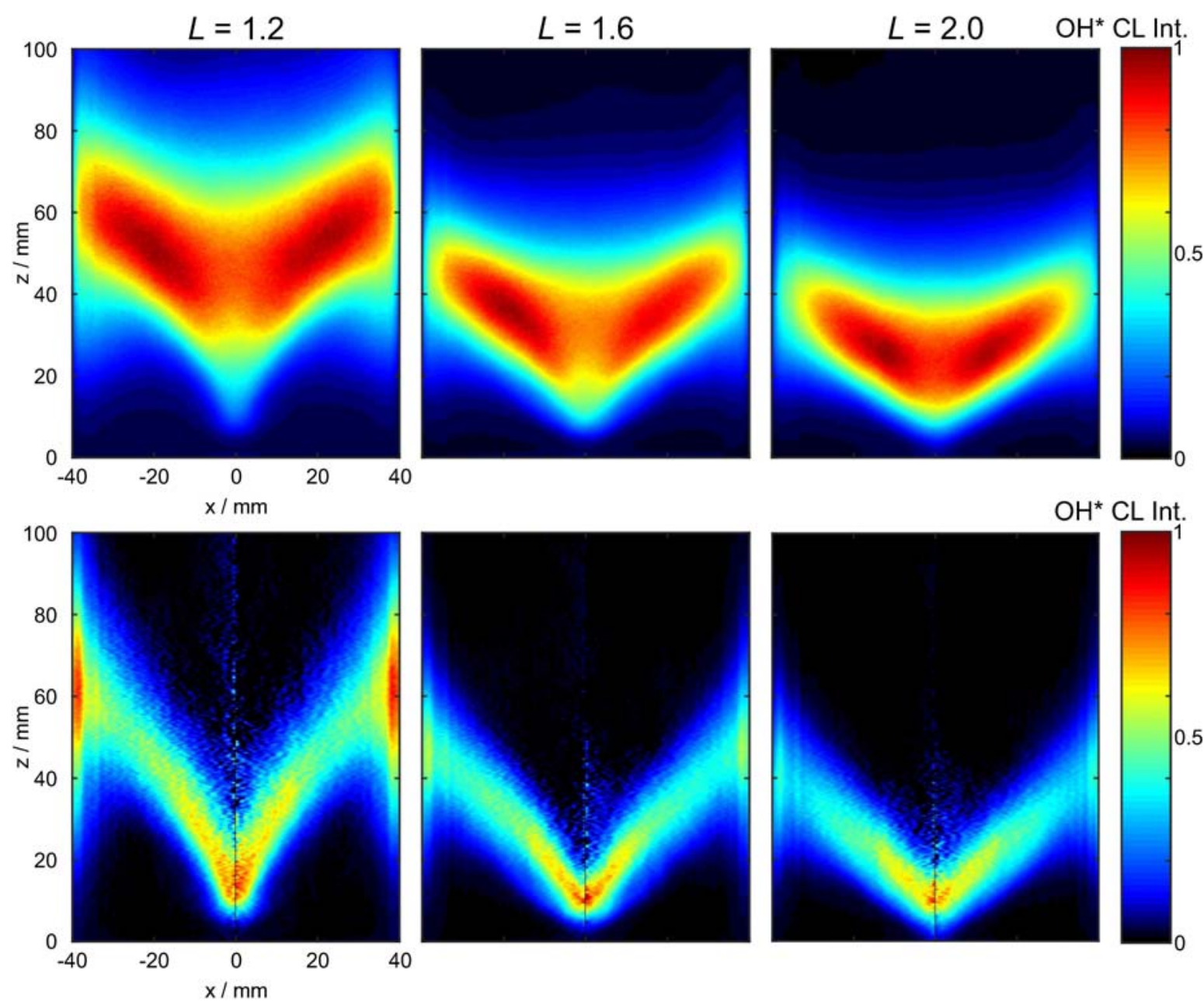

Figure 5. Mean (top row) and Abel-deconvolution (bottom row) of the normalized $\mathrm{OH}^{*}$ chemiluminescence intensity for different air split ratios $L$.

With increasing air split ratio, the flames become more compact, and the opening angle of the flames increases. While for $L=1.2$, where the bulk flow velocity in the inner nozzle is highest, reactions, as visualized by the mean $\mathrm{OH}^{*}$ chemiluminescence intensity, can be observed within the complete combustion chamber (i.e. up to $\mathrm{z}=110 \mathrm{~mm}$ ). For the highest examined air split ratio of $L=2.0$, where twice as much air enters the combustion chamber through the outer nozzle in comparison to the inner nozzle, the flame zone extends up to axial locations $\mathrm{z}<75 \mathrm{~mm}$ and thus is approximately $35 \mathrm{~mm}$ shorter in comparison to $L=1.2$.

Figure 6 shows the average flow field in the non-reacting case for the examined air split ratios. Color coded is the mean total velocity magnitude $|\bar{v}|=\left(\bar{u}^{2}+\bar{v}^{2}+\bar{w}^{2}\right)^{1 / 2}$, and overlaid are the streamlines. The recirculation zone is indicated by the white iso-line of zero axial velocity. The flow field consists of a pronounced inner (IRZ) and outer recirculation zone (ORZ), divided by the inflow, where the highest velocity magnitudes can be observed. With increasing air split ratio $L$, the opening angle of the inflow increases while the peak velocities in the inflow decrease. The axial extension of the IRZ decreases with increasing air split ratio, while the radial extension increases. The axial and radial extension of the outer recirculation zone decreases. Furthermore, the center of the ORZ shifts upstream with increasing air split ratio. 


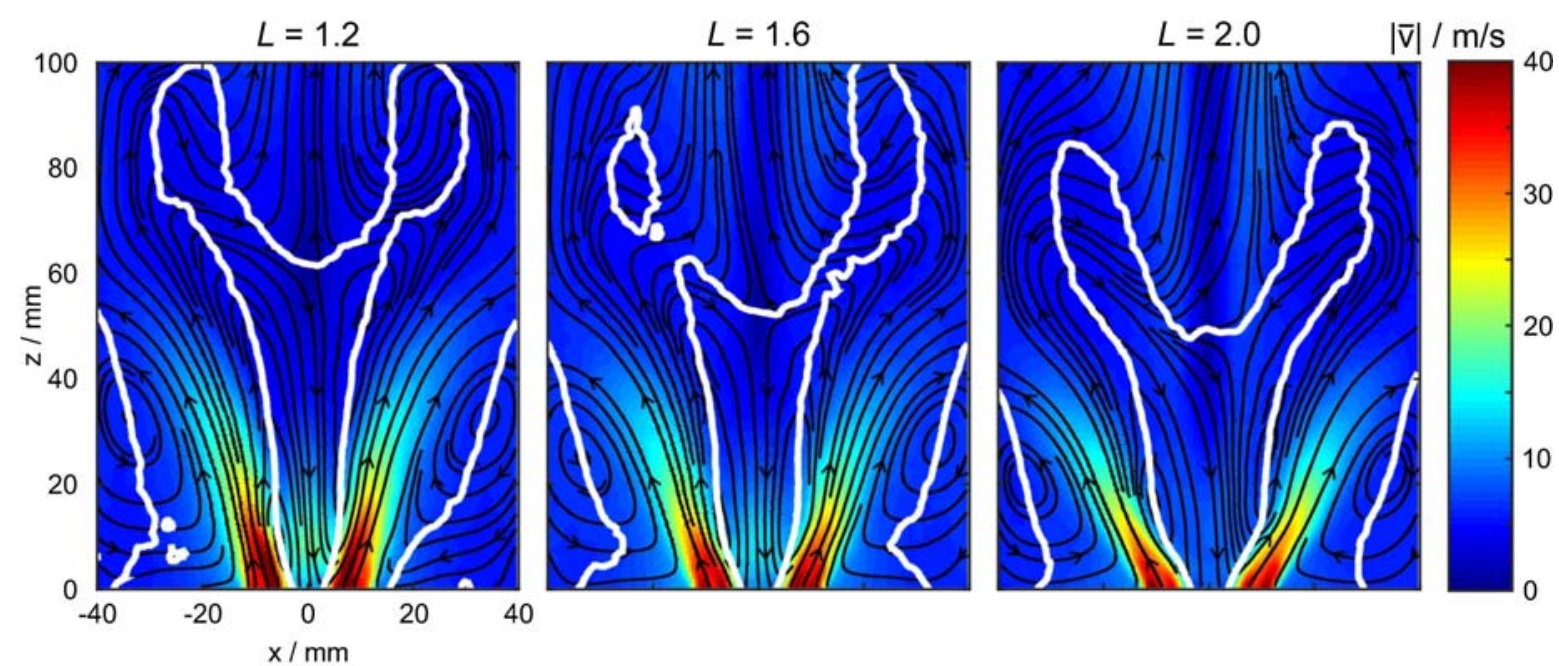

Figure 6. Average non-reacting flow field for different air split ratios $L$. Color coded is the mean velocity magnitude. Overlaid are the streamlines, the white iso-contour represents the outline of the recirculation zones, i.e. the iso-contour of zero axial velocity.

A similar behavior can be seen in Figure 7, which shows the flow field for the same flow conditions, but for the reacting case. Similar to the non-reacting case, the opening angle of the inflow and the size of the inner recirculation zone increases with increasing air split ratio. However, the opening angle of the inflow is significantly larger in the reacting case, while the velocity magnitude at the inflow is comparable for both the reacting and the non-reacting case. In comparison to the non-reacting case, the position of the ORZ and IRZ is shifted upstream. For example, for an air split ratio of $L=1.6$, the center of the ORZ is at $\mathrm{z}=25 \mathrm{~mm}$ for the non-reacting case and at $\mathrm{z}=20 \mathrm{~mm}$ for the reacting case. The highest negative axial velocities on the centerline close to the burner nozzle, i.e. at $\mathrm{z}=2.5 \mathrm{~mm}$ (which is the farthest upstream location at which reliable velocity profiles could be extracted) are observed for $L=1.2$, where axial velocities of up to $\bar{u}=-11.5 \mathrm{~m} / \mathrm{s}$ can be observed. For $L=1.6$, which corresponds to equal bulk flow through the inner and outer nozzle, the minimum observed axial velocities at $\mathrm{z}=2.5 \mathrm{~mm}$ where only $\bar{u}=-8.5 \mathrm{~m} / \mathrm{s}$. A further increase of the air split ratio to $L=2.0$ leads again to an increase in the recirculation zone strength with minimum axial velocities of $\bar{u}=-10.5 \mathrm{~m} / \mathrm{s}$ at $\mathrm{z}=2.5 \mathrm{~mm}$.

The standard deviations of the velocity fields show pronounced shear layers (visualized by the regions of high velocity fluctuations) between the inflow and the IRZ and the inflow and the ORZ: With increasing air split ratio $L$ the strength of the shear layer between the IRZ and the inflow decreases, while the strength of the shear layer between the ORZ and the inflow increases. For $L=2.0$, the shear layer at the ORZ is attached to the nozzle exit, and broadens downstream of the nozzle. Furthermore, a region of high velocity fluctuations can be seen in the lower part of the IRZ. This region is most pronounced for the lowest examined air split ratio $L=1.2$ and least pronounced for the highest examined air split ratio. This region of high velocity fluctuations in the flame stabilization region could be the reason for the observed decrease of the height of the flame root with increasing air split ratio $L$. Furthermore, the tendency of the shear layers at the IRZ to be less pronounced with higher air split ratio might support flame stabilization in this shear layer region and thus could be responsible for the observed trend of decreasing flame length with increasing air split ratio. 

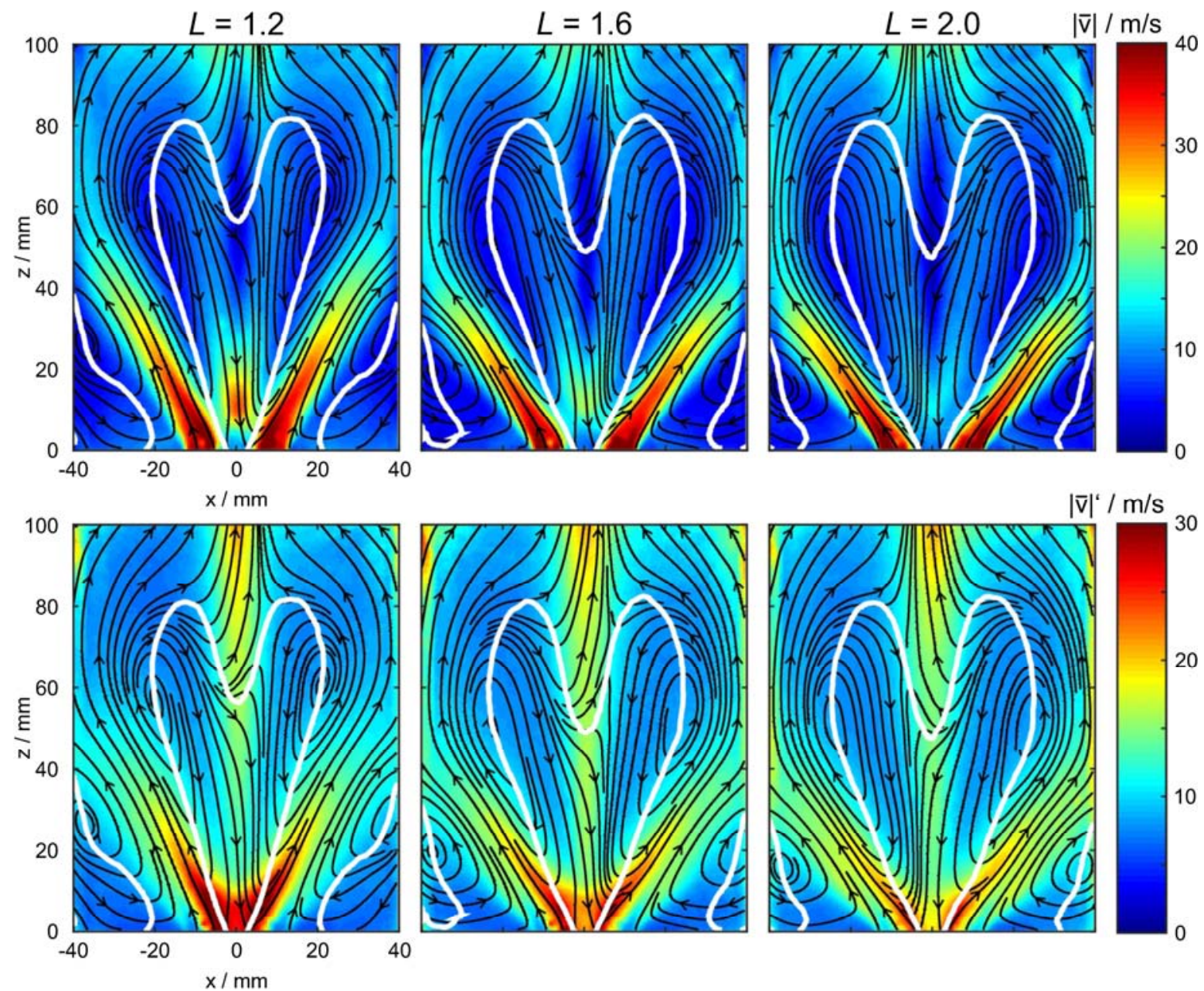

Figure 7. Average (top row) and standard deviation (bottom row) of the reacting flow field for different air split ratios $L$. Color coded is the mean velocity magnitude and the standard deviation of the velocity magnitude, respectively. Overlaid are the streamlines, the white iso-contour represents the outline of the recirculation zones, i.e. the iso-contour of zero axial velocity.

An overview of the acoustic behavior of the different non-reacting flows at different air split ratios is given in Figure 8. Several frequency peaks are observable at all microphone probe locations. One mode is visible for all microphone probe positions, and is amplified when performing a frequency analysis of the difference signal between two microphone probes that are attached on opposite sides of the combustion chamber (labeled "M2-M1" in Figure 8). This mode, which is between approximately $900 \mathrm{~Hz}$ and $1250 \mathrm{~Hz}$ (depending on the air split ratio) is marked as " $\mathrm{f}_{\mathrm{PVC}}$ " and can be associated with a hydro-dynamic instability, a so-called precessing vortex core (PVC). This purely hydro-dynamic instability consists of a tornado-like vortex structure that precesses around the combustion chamber at a given frequency. 
$L=1.2$

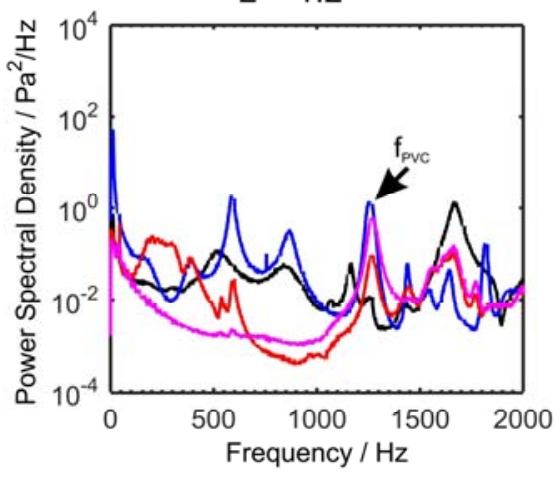

$L=1.6$

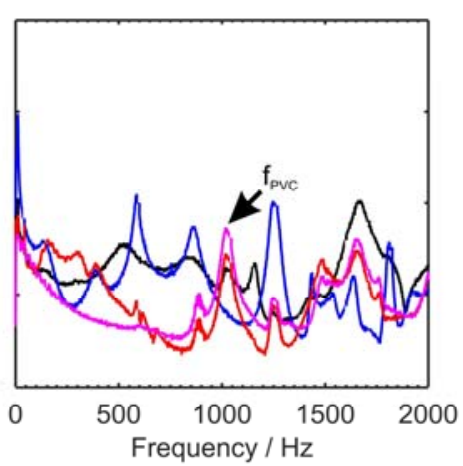

$L=2.0$
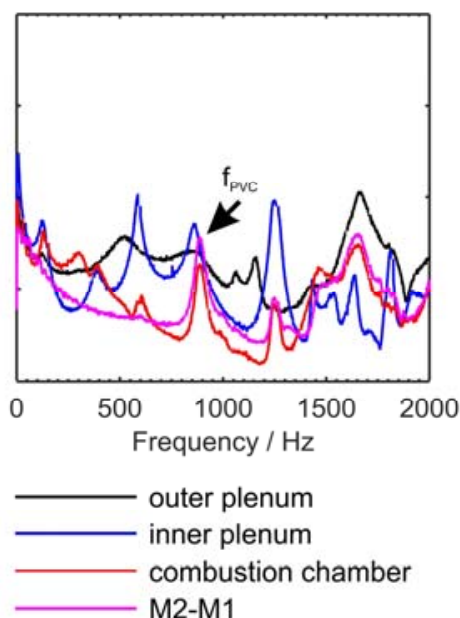

Figure 8. Acoustic spectra of the combustion chamber, inner plenum and outer plenum for the non-reacting operating conditions.

A similar behavior can be seen in Figure 9, which shows the frequency spectra for the corresponding reacting case. Here as well several frequency peaks can be observed. However, the amplitudes of those frequency peaks are small in comparison to flames with thermo-acoustic instabilities in the same combutor ${ }^{23}$, and no pronounced selfexcited thermo-acoustic pulsations were present in the combustor for all here examined operating conditions. The frequency peak at approximately $190 \mathrm{~Hz}$, which can be seen for all air split ratios $L$ is associated with a weak thermo-acoustic mode that corresponds to a Helmholtz-mode between the outer air plenum and the combustion chamber $^{23}$. For the reacting case, the frequency of the PVC is between approximately $1000 \mathrm{~Hz}$ and $1350 \mathrm{~Hz}$ (depending on the air split ratio), or approximately $10 \%$ higher than in the non-reacting case.
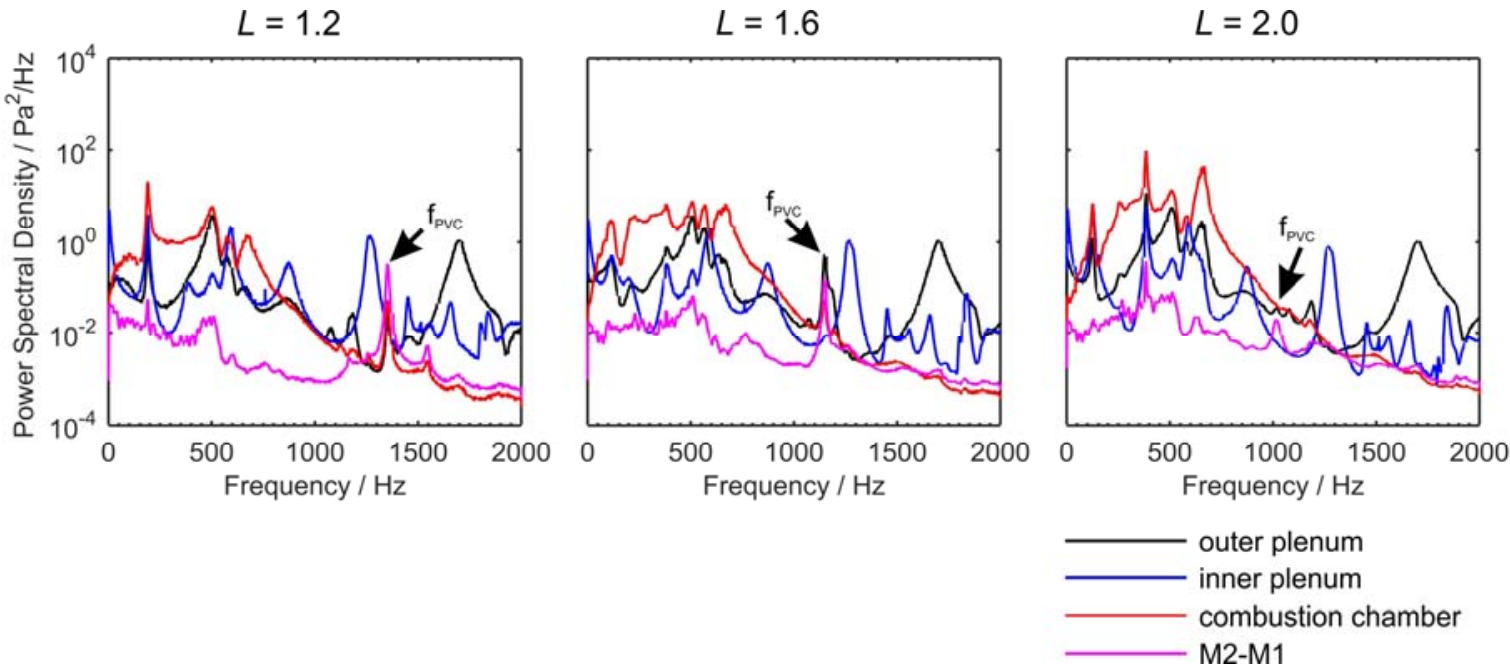

Figure 9. Acoustic spectra of the combustion chamber, inner plenum and outer plenum for the reacting operating conditions.

In several swirl flow configurations, precessing vortex cores (PVCs) were observed in the non-reacting and / or reacting flow field under different operating conditions ${ }^{2,14-21,35-37}$. The here examined burner featured a PVC for all examined operating conditions. This flow structure can be observed in the acoustic spectra of the combustion chamber due to its asymmetric shape and furthermore can influence the heat release distribution significantly.

An overview of the measured PVC frequencies for the here examined operating conditions and the corresponding Strouhal numbers is given in Table 2. The Strouhal number $S r$, which is a dimensionless number for instationary flows, is defined as $S r=(f \cdot l) / u$. Here, $\mathrm{f}$ is a frequency, $l$ a characteristic length scale of the flow 
and $u$ the flow velocity. For the current configuration, the diameter of the inner air nozzle $\left(\mathrm{D}_{\mathrm{i}}=15 \mathrm{~mm}\right)$ was chosen as characteristic length scale and the bulk flow velocity through the inner air nozzle (before the fuel injection) as characteristic flow velocity. Both non-reacting and reacting case feature an almost constant Strouhal number for the measured PVC frequency in dependence of the bulk flow velocity in the inner nozzle. The small differences in Strouhal numbers between the operating conditions can be explained by the mechanism of the fuel injection. In the current configuration, fuel is injected into the swirling inner air flow through several small holes in the inner air nozzle, upstream of the burner exit plane. Thus, due to momentum conservation, the rotationary momentum of the air flow is decreased by the non-swirling fuel, and this decrease in rotationary momentum also influences the PVC frequency. Furthermore, this effect is stronger pronounced at high air split ratios, where the ratio of air to fuel in the inner nozzle is the lowest.

\begin{tabular}{|c|c|c|c|c|c|c|}
\hline $\begin{array}{c}L \\
{[-]}\end{array}$ & $\begin{array}{l}\dot{\mathrm{m}}_{\mathrm{air}, \mathrm{i}} \\
{[\mathrm{g} / \mathrm{min}]}\end{array}$ & $\begin{array}{c}\dot{\mathrm{m}}_{\mathrm{air}, \mathrm{o}} \\
{[\mathrm{g} / \mathrm{min}]}\end{array}$ & $\begin{array}{c}\mathrm{f}_{\mathrm{PVC}, \mathrm{nr}} \\
{[\mathrm{Hz}]}\end{array}$ & $\begin{array}{c}\mathrm{Sr}_{\mathrm{nr}} \\
{[-]}\end{array}$ & $\begin{array}{l}\mathrm{f}_{\mathrm{PVC}, \mathrm{r}} \\
{[\mathrm{Hz}]}\end{array}$ & $\begin{array}{l}\mathrm{Sr}_{\mathrm{r}} \\
{[-]}\end{array}$ \\
\hline 1.2 & 334 & 401 & 1254 & 0.77 & 1351 & 0.83 \\
\hline 1.6 & 282 & 451 & 1017 & 0.74 & 1148 & 0.84 \\
\hline 2.0 & 245 & 490 & 888 & 0.75 & 1013 & 0.85 \\
\hline
\end{tabular}

Table 2. Measured PVC frequencies for the non-reacting and reacting case and the corresponding Strouhal numbers Sr.

Of further interest is the precession frequency of the PVC for a multitude of operating conditions and whether or not the before observed trend of constant Strouhal numers is representative for other operating conditions. In previous configurations, it was observed that the PVC scales linearly with the total air flow rate; however in previous configurations, the air flow through the inner and outer air nozzle could not be controlled separately. Figure 10 shows the PVC precession frequency for a variety of operating conditions (thermal power, equivalence ratio and air split ratio) for the non-reacting (blue) and reacting (red) case versus the air mass flow through the inner air nozzle. For all examined operating conditions, the PVC frequency scales linearly with the air flow ratio through the inner nozzle, with a constant Strouhal number of $\mathrm{Sr}_{\mathrm{nr}}=0.78$ for the non-reacting case and $\mathrm{Sr}_{\mathrm{r}}=0.85$ for the reacting case, while it is almost independent of the air flow through the outer nozzle.

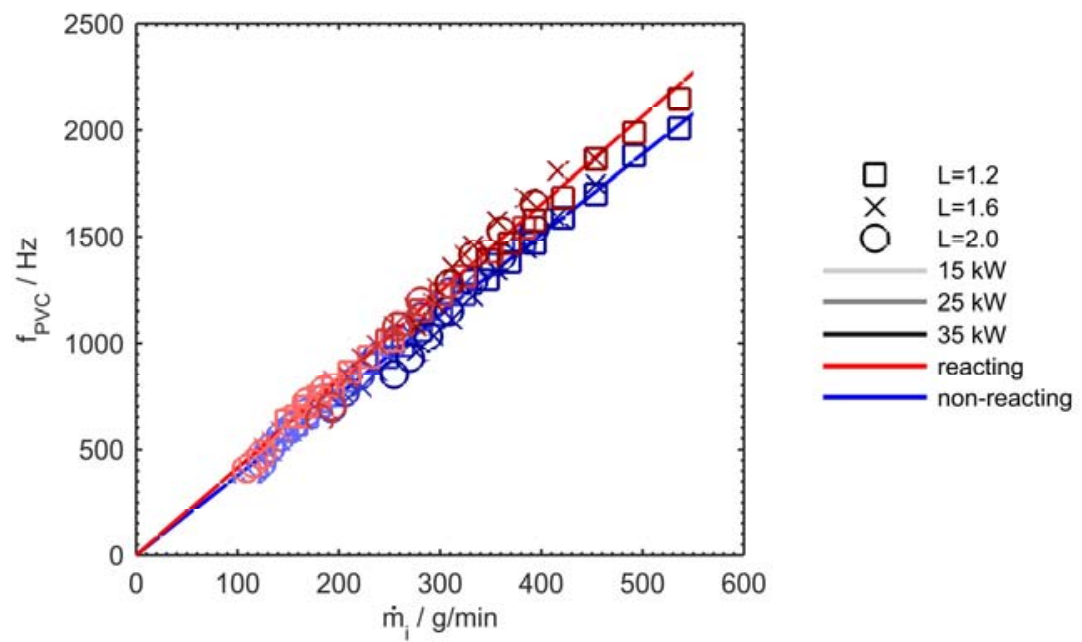

Figure 10. Dependence of the PVC frequency on the air mass flow through the inner nozzle for the reacting (red) and non-reacting (blue) case.

Small deviations from a strictly linear trend stem, as mentioned above, from the fuel injection into the inner air stream. 


\section{Precessing Vortex Core (PVC) Dynamics and Influence on the Flame}

A precessing vortex core (PVC) was present for all examined operating conditions, and in the following section, a brief overview of the influence of the PVC on the heat release distribution in the combustion chamber will be provided. Figure 11 shows an exemplary image sequence of the $\mathrm{OH}^{*}$ chemiluminescence distribution in the combustion chamber, phase averaged on the phase angle $\alpha$ of the PVC. The phase angle of the PVC was determined from proper orthogonal decomposition of the simultaneously acquired velocity fields according to the method outlined by Stöhr et al. ${ }^{6}$.
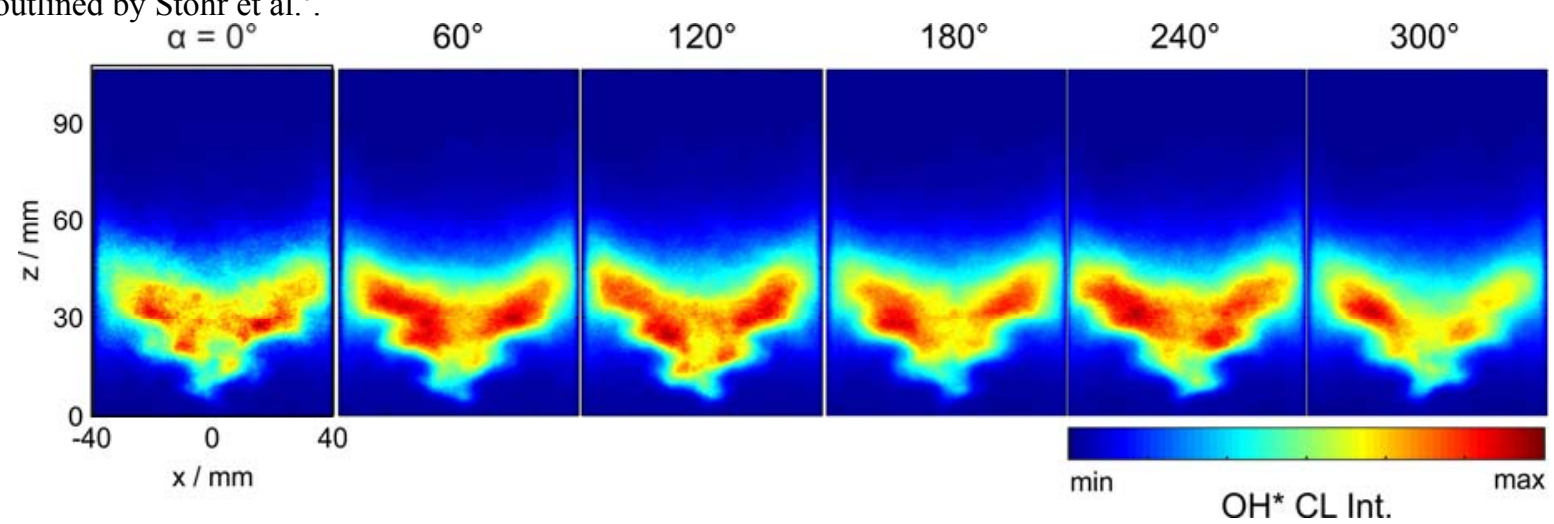

Figure 11. Phase-averaged $\mathrm{OH}^{*}$ chemiluminescence distribution in the combustion chamber to demonstrate the influence of the PVC on the heat release dynamics at $L=1.6$. Color coded is the $\mathrm{OH}^{*}$ chemiluminescence intensity.

While the total $\mathrm{OH}^{*}$ chemiluminescence does not vary significantly between the different phase angles, the shape of the heat release zone shows a strong dependency on the phase angle. On contrast to the long-time averages presented before, the structure of the phase-averaged heat release zone is asymmetric and follows the zic-zac pattern of the precessing vortex core. The flame root precesses around the combustion chamber with the frequency of the PVC, thus also having a non-stationary anchoring point with respect to the PVC phase angle. From the here presented results, it can be concluded that the PVC not only has a strong influence on the flow field and acoustic modes in the combustion chamber, but also on the instantaneous distribution of the heat release zone.

\section{Conclusions}

A dual-swirl gas turbine model combustor (GTMC) was studied with detailed laser-based and optical techniques. The burner featured two separate air plenums for each air flow, such that the air split ratio between the inner and the outer air flow can be set exactly. A set of operating conditions with different air split ratios, all of which exhibited no pronounced self-excited thermo-acoustic oscillations, but a hydro-dynamic instability, a so-called precessing vortex core, were studied with detailed laser-baser and optical diagnostics. The flame shape was measured by chemiluminescence imaging and the three-component planar flow-field by stereoscopic Particle Image Velocimetry. Furthermore, the simultaneous major species concentration, mixture fraction, and temperature was measured by laser Raman scattering for the reference operating condition, which featured equal pressure loss across both swirlers. The air split ratio had a major impact, both on the non-reacting and reacting flow field as well as on the flame shape. With increasing air split ratio, the opening angle of the inflow increased and the length of the inner recirculation zone decreased for the non-reacting case. In the reacting case, the length of the inner recirculation zone remained more or less constant for all examined operating conditions. For both the non-reacting and reacting case, the radial extend of the inner recirculation zone increased with increasing air split ratio. The flame length decreased and the flame stabilized closer to the burner exit plane significantly with increasing air split ratio. The frequency of the PVC was at a constant Strouhal number, based on the air mass flow through the inner fuel nozzle, and was independent of the air split ratio. The Strouhal number for the PVC was $\mathrm{Sr}_{\mathrm{nr}}=0.78$ for the non-reacting case and $\mathrm{Sr}_{\mathrm{r}}=0.85$ for the reacting case.

\section{Acknowledgments}

The financial support by the German Research Council (DFG) within the Collaborative Research Center 606 is gratefully acknowledged. 


\section{References}

${ }^{1}$ Arndt, C. M., Steinberg, A. M., Boxx, I. G., Meier, W., Carter, C. D., and Aigner, M. "Flow-Field and Flame Dynammics of a Gas Turbine Model Combustor During Transition Between Thermo-Acoustically Stable und Unstable States," Proceedings of the ASME Turbo Expo. 2010, GT2010-22830.

${ }^{2}$ Oberleithner, K., Stöhr, M., Im, S. H., Arndt, C. M., and Steinberg, A. M., "Formation and flame-induced suppression of the precessing vortex core in a swirl combustor: Experiments and linear stability analysis," Combustion and Flame, Vol. 162, No. 8, 2015, pp. 3100-3114.

${ }^{3}$ Candel, S., Durox, D., Schuller, T., Bourgouin, J.-F., and Moeck, J. P., "Dynamics of Swirling Flames," Annual Review of Fluid Mechanics, Vol. 46, 2014, pp. 147-173.

${ }^{4}$ Caux-Brisebois, V., Steinberg, A. M., Arndt, C. M., and Meier, W., "Thermo-acoustic velocity coupling in a swirl stabilized gas turbine model combustor," Combustion and Flame, Vol. 161, No. 12, 2014, pp. 3166-3180.

${ }^{5}$ Moeck, J. P., Bourgouin, J.-F., Durox, D., Schuller, T., and Candel, S., "Nonlinear interaction between a precessing vortex core and acoustic oscillations in a turbulent swirling flame," Combustion and Flame, Vol. 159, No. 8, 2012, pp. 2650-2688.

${ }^{6}$ Stöhr, M., Boxx, I., Carter, C. D., and Meier, W., "Experimental study of vortex-flame interaction in a gas turbine model combustor," Combustion and Flame, Vol. 159, No. 8, 2012, pp. 2636-2649.

${ }^{7}$ Stöhr, M., Arndt, C. M., and Meier, W., "Transient effects of fuel-air mixing in a partially-premixed turbulent swirl flame," Proceedings of the Combustion Institute, Vol. 35, No. 3, 2015, pp. 3327-3335.

${ }^{8}$ Huang, Y., and Yang, V., "Dynamics and stability of lean-premixed swirl-stabilized combustion," Progress in Energy and Combustion Science, Vol. 35, No. 4, 2009, pp. 293-364.

${ }^{9}$ Galley, D., Ducruix, S., Lacas, F., and Veynante, D., "Mixing and stabilization study of a partially premixed swirling flame using laser induced fluorescence," Combustion and Flame, Vol. 158, No. 1, 2011, pp. 155-171.

${ }^{10}$ Freitag, M., Klein, M., Gregor, M., Geyer, D., Schneider, C., Dreizler, A., and Janicka, J., "Mixing analysis of a swirling recirculating flow using DNS and experimental data," International Journal of Heat and Fluid Flow, Vol. 27, No. 4, 2006, pp. 636-643.

${ }^{11}$ Fröhlich, J., García-Villalba, M., and Rodi, W., "Scalar Mixing and Large-Scale Coherent Structures in a Turbulent Swirling Jet," Flow, Turbulence and Combustion, Vol. 80, No. 1, 2008, pp. 47-59.

${ }^{12}$ Stöhr, M., Arndt, C. M., and Meier, W., "Effects of Damkohler number on vortex-flame interaction in a gas turbine model combustor," Proceedings of the Combustion Institute, Vol. 34, No. 2, 2013, pp. 3107-3115.

${ }^{13}$ Steinberg, A. M., Boxx, I., Stöhr, M., Carter, C. D., and Meier, W., "Flow-flame interactions causing acoustically coupled heat release fluctuations in a thermo-acoustically unstable gas turbine model combustor," Combustion and Flame, Vol. 157, No. 12, 2010, pp. 2250-2266.

${ }^{14}$ Anacleto, P. M., Fernandes, E. C., Heitor, M. V., and Shtork, S. I., "Swirl Flow Structure and Flame Characteristics in a Model Lean Premixed Combustor," Combustion Science and Technology, Vol. 175, No. 8, 2003, pp. 1369-1388.

${ }^{15}$ Fokaides, P., Weiß, M., Kern, M., and Zarzalis, N., "Experimental and Numerical Investigation of Swirl Induced SelfExcited Instabilities at the Vicinity of an Airblast Nozzle," Flow, Turbulence and Combustion, Vol. 83, 2009, pp. 511-533.

${ }^{16}$ Patel, N., and Menon, S., "Simulation of spray-turbulence-flame interactions in a lean direct injection combustor," Combustion and Flame, Vol. 153, No. 1-2, 2008, pp. 228-257.

${ }^{17}$ Schneider, C., Dreizler, A., and Janicka, J., "Fluid Dynamical Analysis of Atmospheric Reacting and Isothermal Swirling Flows," Flow Turbulence and Combustion, Vol. 74, 2005, pp. 103-127.

${ }^{18}$ Schildmacher, K.-U., Koch, R., and Bauer, H.-J., "Experimental Characterization of Premixed Flame Instabilities of a Model Gas Turbine Burner," Flow, Turbulence and Combustion, Vol. 76, 2006, pp. 177-197.

${ }^{19}$ De, A., Zhu, S., and Acharya, S., "An Experimental and Computational Study of a Swirl-Stabilized Premixed Flame," Journal of Engineering for Gas Turbines and Power, Vol. 132, No. 7, 2010, p. 071503.

${ }^{20}$ Freitag, M., and Janicka, J., "Investigation of a strongly swirled unconfined premixed flame using LES," Proceedings of the Combustion Institute, Vol. 31, No. 1, 2007, pp. 1477-1485.

${ }^{21}$ Selle, L., Lartigue, G., Poinsot, T., Koch, R., Schildmacher, K.-U., Krebs, W., Prade, B., Kaufmann, P., and Veynante, D., "Compressible large eddy simulation of turbulent combustion in complex geometry on unstructured meshes," Combustion and Flame, Vol. 137, No. 4, 2004, pp. 489-505.

${ }^{22}$ Boxx, I., Arndt, C. M., Carter, C. D., and Meier, W., "Highspeed Laser Diagnostics for the Study of Flame Dynamics in a Lean Premixed Gas Turbine Model Combustor," Experiments in Fluids, Vol. 52, No. 3, 2012, pp. 555-567.

${ }^{23}$ Arndt, C. M., Severin, M., Dem, C., Stohr, M., Steinberg, A. M., and Meier, W., "Experimental analysis of thermo-acoustic instabilities in a generic gas turbine combustor by phase-correlated PIV, chemiluminescence, and laser Raman scattering measurements," Experiments in Fluids, Vol. 56, No. 4, 2015, p. 69.

${ }^{24}$ Meier, W., Dem, C., and Arndt, C. M., "Mixing and reaction progress in a confined swirl flame undergoing thermo-acoustic oscillations studied with laser Raman scattering," Experimental Thermal and Fluid Science, Vol. 73, 2015, pp. 71-78.

${ }^{25}$ Bärow, E., Koch, R., and Bauer, H.-J. "Comparison of oscillation modes of spray and gaseous flames," Proceedings of the Eighth Mediterranean Combustion Symposium. 2013, EGTSC-26.

${ }^{26}$ Kraus, C., and Bockhorn, H. "Experimental and numerical investigation of combustion instabilities in swirl-stabilized flames operated in partially-premixed mode," Proceedings of the European Combustion Meeting. 2013, P5-26.

${ }^{27}$ Kraus, C., Harth, S., and Bockhorn, H., "Experimental investigation of combustion instabilities in lean swirl-stabilized partially-premixed flames in single- and multiple-burner setup," International Journal of Spray and Combustion Dynamics, Vol. 8, No. 1, 2016, pp. 4-26. 
${ }^{28}$ Kraus, C., Selle, L., Poinsot, T., Arndt, C. M., and Bockhorn, H., "Influence of Heat Transfer and Material Temperature on Combustion Instabilities in a Swirl Burner," Journal of Engineering for Gas Turbines and Power, Vol. 139, No. 5, 2016, p. 051503 .

${ }^{29}$ Lawson, N. J., and Wu, J., "Three-dimensional particle image velocimetry: experimental error analysis of a digital angular stereoscopic system," Measurement Science and Technology, Vol. 8, No. 12, 1997, pp. 1455-1464.

${ }^{30}$ Keck, O., Meier, W., Stricker, W., and Aigner, M., "Establishment of a Confined Swirling Natural Gas/Air Flame as a Standard Flame: Temperature and Species Distributions from Laser Raman Measurements," Combustion Science and Technology, Vol. 174, No. 8, 2002, pp. 117-151.

${ }^{31}$ Meier, W., Weigand, P., Duan, X. R., and Giezendanner-Thoben, R., "Detailed characterization of the dynamics of thermoacoustic pulsations in a lean premixed swirl flame," Combustion and Flame, Vol. 150, No. 1-2, 2007, pp. 2-26.

${ }^{32}$ Bergmann, V., Meier, W., Wolff, D., and Stricker, W., "Application of spontaneous Raman and Rayleigh scattering and 2D LIF for the characterization of a turbulent $\mathrm{CH}_{4} / \mathrm{H}_{2} / \mathrm{N}_{2}$ jet diffusion flame," Applied Physics B, Vol. 66, No. 4, 1998, pp. 489-502.

${ }^{33}$ Bilger, R. W., and Starner, S. H., "On reduced mechanisms for methane/air combustion in nonpremixed flames," Combustion and Flame, Vol. 80, No. 2, 1990, pp. 135-149.

${ }^{34}$ Duan, X. R., Meier, W., Weigand, P., and Lehmann, B., "Phase-Resolved Laser Raman Scattering and Laser Doppler Velocimetry Applied to Periodic Instabilities in a Gas Turbine Model Combustor," Applied Physics B, Vol. 80, 2005, pp. 389396.

${ }^{35}$ Terhaar, S., Oberleithner, K., and Paschereit, C. O., "Key parameters governing the precessing vortex core in reacting flows: An experimental and analytical study," Proceedings of the Combustion Institute, Vol. 35, No. 3, 2015, pp. 3347-3354

${ }^{36}$ Duwig, C., and Fuchs, L., "Large eddy simulation of vortex breakdown/flame interaction," Physics of Fluids, Vol. 19, 2007, p. 075103.

${ }^{37}$ Steinberg, A. M., Arndt, C. M., and Meier, W., "Parametric study of vortex structures and their dynamics in swirl-stabilized combustion," Proceedings of the Combustion Institute, Vol. 34, No. 2, 2013, pp. 3117-3125. 